\title{
Stringently Defined Otitis Prone Children Demonstrate Deficient Naturally Induced Mucosal Antibody Response to Moraxella catarrhalis Proteins
}

\author{
Dabin Ren ${ }^{1 *}$, Timothy F. Murphy ${ }^{2}$, Eric R. Lafontaine ${ }^{3}$ and Michael E. Pichichero ${ }^{1}$ \\ ${ }^{1}$ Rochester General Hospital Research Institute, Rochester, NY, United States, ${ }^{2}$ Clinical and Translational Research Center, \\ Jacobs School of Medicine and Biomedical Sciences, University at Buffalo, The State University of New York, Buffalo, NY, United \\ States, ${ }^{3}$ Department of Infectious Diseases, College of Veterinary Medicine, University of Georgia, Athens, GA, United States
}

OPEN ACCESS

Edited by:

Yasmin Thanavala,

Roswell Park Cancer Institute,

United States

Reviewed by:

Pablo Daniel Becker,

King's College London,

United Kingdom

Charles Kelly,

King's College London,

United Kingdom

*Correspondence:

Dabin Ren

dabin.ren@rochesterregional.org

Specialty section:

This article was submitted

to Mucosal Immunity,

a section of the journal

Frontiers in Immunology

Received: 08 June 2017

Accepted: 26 July 2017

Published: 11 August 2017

Citation:

Ren D, Murphy TF, Lafontaine ER and Pichichero ME (2017) Stringently Defined Otitis Prone Children

Demonstrate Deficient Naturally Induced Mucosal Antibody Response to Moraxella catarrhalis Proteins.

Front. Immunol. 8:953. doi: 10.3389/fimmu.2017.00953
Moraxella catarrhalis (Mcat) is a prominent mucosal pathogen causing acute otitis media (AOM). We studied Mcat nasopharyngeal (NP) colonization, AOM frequency and mucosal antibody responses to four vaccine candidate Mcat proteins: outer membrane protein (OMP) CD, oligopeptide permease (Opp) A, hemagglutinin (Hag), and Pilin A clade 2 (PilA2) from stringently defined otitis prone (sOP) children, who experience the greatest burden of disease, compared to non-otitis prone (NOP) children. sOP children had higher NP colonization of Mcat (30 vs. 22\%, $P=0.0003$ ) and Mcat-caused AOM rates (49 vs. $24 \%, P<0.0001$ ) than NOP children. Natural acquisition of mucosal antibodies to Mcat proteins OMP CD (lgG, $P<0.0001)$, OppA (lgG, $P=0.018)$, Hag (lgG and $\lg A$, both $P<0.0001)$, and PilA2 (IgA, $P<0.0001)$ was lower in SOP than NOP children. Higher levels of mucosal lgG to Hag $(P=0.039)$ and PilA2 $(P=0.0076)$, and lgA to OMP CD $(P=0.010)$, OppA $(P=0.030)$, and PilA2 $(P=0.043)$ were associated with lower carriage of Mcat in NOP but not sOP children. Higher levels of mucosal IgG to OMP $\mathrm{CD}(P=0.0070)$ and $\mathrm{Hag}(P=0.0003)$, and $\operatorname{lgA}$ to $\mathrm{Hag}(P=0.0067)$ at asymptomatic colonization than those at onset of $A O M$ were associated with significantly lower rate of Mcat NP colonization progressing to AOM in NOP compared to sOP children ( 3 vs. $26 \%$, $P<0.0001$ ). In conclusion, sOP children had a diminished mucosal antibody response to Mcat proteins, which was associated with higher frequencies of asymptomatic NP colonization and NP colonization progressing to Mcat-caused AOM. Enhancing Mcat antigen-specific mucosal immune responses to levels higher than achieved by natural exposure will be necessary to prevent $\mathrm{AOM}$ in SOP children.

Keywords: otitis prone, nasopharyngeal colonization, acute otitis media, immunogenicity, recombinant proteins, antigen, mucosal immune response, carriage

Abbreviations: Mcat, Moraxella catarrhalis; AOM, acute otitis media; OP, otitis prone; sOP, stringently defined otitis prone; NOP, non-otitis prone; NP, nasopharyngeal; OMP, outer membrane protein; Spn, Streptococcus pneumoniae; NTHi, nontypeable Haemophilus influenzae; OppA, oligopeptide permease A; MID, Moraxella IgD-binding protein; Hag, hemagglutinin; TM, tympanic membrane; Msp, Moraxella surface protein; PilA2, Pilin A clade 2; MEF, middle ear fluid; ICH, International Council for Harmonization; GM, geometric mean; CI, confidence interval; OME, otitis media with effusion; URI, upper respiratory infection; sIgA, secretory IgA. 


\section{INTRODUCTION}

Acute otitis media (AOM) is the most common infectious disease among children to cause parents to seek medical care for their child and receive antibiotics. Moraxella catarrhalis (Mcat) has been ranked as the third most common cause of AOM after Streptococcus pneumoniae (Spn) and non-typeable Haemophilus influenzae (NTHi) in children (1). However, the virulence of Mcat as an otopathogen may be increasing as evidenced by an increased occurrence of tympanic membrane (TM) rupture caused by the organism (Pichichero, unpublished observation). Our recent studies on the prevalence of otopathogens show that Mcat has overtaken Spn and NTHi to become the most frequent cause of episodic and recurrent AOM in children (2). Similarly, Mcat was recently identified as the most common otopathogen in Finish children (3). AOM often recurs and poses a high burden on the quality of life of children, the health care system and the economy worldwide (4).

There is no licensed vaccine for Mcat, and the development of an Mcat vaccine is currently moving from animal studies toward clinical trials. A number of antigens have been identified as potential Mcat vaccine candidates, of which outer membrane protein (OMP) $\mathrm{CD}$, oligopeptide permease (Opp) A, hemagglutinin (Hag), and Pilin A clade 2 (PilA2) are promising representatives. OMP CD is a porin and adhesin and is highly conserved with exposed epitopes on the bacterial surface $(5,6)$. OppA is a highly conserved oligopeptide binding protein mediating the uptake of peptides and fitness of the organism in the respiratory tract $(7,8)$. Hag, also known as Moraxella IgD-binding protein (MID), is an adhesin, Hag, and autotransporter containing surface exposed and conserved epitopes (9). PilA2 is a conserved pilin involved in natural genetic transformation, biofilm formation, and adherence of the bacteria to human epithelial cells (10-12).

Otitis prone (OP) defines a health status of children who have recurrent AOM, with at least three episodes in 6 months or four episodes in a 12-month time span $(13,14)$. To meet the definition of stringently defined otitis prone (sOP), a child must have every episode proven by a tympanocentesis-derived middle ear fluid (MEF) positive culture of an otopathogen while non-otitis prone (NOP) children are those with $0-2$ episodes of AOM per year $(14,15)$. Because sOP children are most vulnerable to AOM, they should be the focused population of vaccination against AOM. Evaluating naturally induced humoral immune responses to $M c a t$ proteins may disclose the immunogenicity of these antigens and functional activity of the antibodies elicited by these proteins in the targeted age and most vulnerable population, especially sOP children. We have found that during nasopharyngeal (NP) colonization by Mcat all four antigens, OMP CD, OppA, Hag, and PilA2, are immunogenic in both sOP and NOP children (16). The age-dependent increase of naturally induced serum antibody ranked as OppA $>$ Hag5-9 > OMP CD $>$ PilA2 in both sOP and NOP children (Ren et al., unpublished). We also found that $\mathrm{SOP}$ children have deficient production of serum antibodies to OMP CD, OppA, Hag, and PilA2 at asymptomatic Mcat NP colonization and onset of AOM (Ren et al., unpublished), similar to our findings for protein vaccine candidates of Spn (17) and NTHi (18).
Moraxella catarrhalis is a mucosal pathogen and mucosal immunity plays an important role in host defense against Mcat infections. Here, we studied Mcat NP colonization, AOM frequency and mucosal antibody responses to four vaccine candidate Mcat proteins, OMP CD, OppA, Hag, and PilA2 in nasal washes from sOP compared to NOP children to identify differences in pathogenicity and mucosal immune responses.

\section{MATERIALS AND METHODS}

\section{Subjects and Sampling}

The samples collected and analyzed were obtained during a prospective study supported by the National Institute of Deafness and Communication Disorders, as previously described $(19,20)$. Healthy children without previous episodes of AOM were enrolled at 6 months of age from a middle class, suburban sociodemographic pediatric practice in Rochester, NY, USA during years 2006-2016. For this study, we assessed a total of 628 children followed prospectively until 36 months of age. NP and oropharyngeal cultures were obtained seven times during the study period at $6,9,12,15,18,24$, and 30-36 months of age. Whenever children experienced an AOM episode, a confirmatory tympanocentesis was performed, and MEF was microbiologically assessed (an essential component to the definition of "stringently defined" AOM since virtually all prior studies have relied on only a clinical diagnosis that is known to be variably accurate). NP, oropharyngeal, and MEF sampling was conducted as previously described $(19,21)$, and the samples were analyzed to identify bacterial pathogens by using standard culture and PCR assay (19). The study was approved by the Rochester General Hospital Research Subjects Review Boards. Written informed consent was obtained for participation, and all procedures are in accordance with the Declaration of Helsinki.

Nasal wash samples were obtained by instilling $1 \mathrm{ml}$ of sterile phosphate-buffered saline (PBS) into each nare and then aspirating from each nare yielding approximately $2 \mathrm{ml}$ of material for each subject. The nasal wash solution was subsequently centrifuged at $3,000 \mathrm{rpm}(1,100 \times \mathrm{g})$ at $4^{\circ} \mathrm{C}$ for $10 \mathrm{~min}$, and the supernatant was then stored at $-80^{\circ} \mathrm{C}$ until used for quantification of mucosal antibody by using enzyme-linked immunosorbent assay (ELISA).

\section{Enzyme-Linked Immunosorbent Assay}

Recombinant Mcat proteins OMP CD, OppA, Hag5-9 (truncated Hag protein), and PilA2 were expressed and purified as previously described $(7,9,11,22)$. Protein-specific antibody concentrations were determined by ELISA using purified recombinant proteins. Three hundred eighty-four well Greiner Microlon plates were coated with $1-3 \mu \mathrm{g} / \mathrm{ml}$ of individual proteins $\left(20 \mu \mathrm{l} /\right.$ well) in PBS (pH 7.4) and incubated at $37^{\circ} \mathrm{C}$ for $1 \mathrm{~h}$. After five washes, the plates were blocked with $10 \%$ fetal bovine serum in PBS (pH 7.4) at room temperature for $1 \mathrm{~h}(40 \mu \mathrm{l} /$ well). After washing, $20 \mu \mathrm{l}$ of nasal wash twofold serially diluted in a buffer of PBS, $0.5 \%$ BSA, with $0.005 \%$ Tween was added to each well. Carimune (CSL Behring AG, Bern, Switzerland) for OMP CD IgG assay and Gammagard (Baxter, Deerfield, IL, USA) for OppA, Hag, and PilA2 IgG assays or in-house human 
sera for IgA assays were used as references and in-house control sera with high and low titers were run on each plate. The plates were incubated at room temperature for $30 \mathrm{~min}$ followed by the addition of affinity-purified goat antihuman IgG/IgA antibody conjugated to horseradish peroxidase (Bethyl Laboratories, Montgomery, TX, USA) as a secondary antibody. The reaction products were developed with TMB Microwell Peroxidase Substrate System (KPL, Gaithersburg, MD, USA), stopped by addition of $1.0 \mathrm{M}$ phosphoric acid and read by a Spectramax 340PC plate reader (Molecular Devices, Sunnyvale, CA, USA) using a 450-nm filter.

To provide quantitative results on antibody concentrations, the level of the specific antibody present in the unknown sample was determined by comparing to an internal reference serum (Carimune for OMP CD IgG and Gammagard for OppA IgG, Hag IgG, and PilA2 IgG or in-house human IgA sera). The levels of IgG and IgA in the reference serum were quantitatively measured by using a human IgG or IgA ELISA quantitation kit (Bethyl laboratories). A four-parameter logistic-log function was used to form the reference and sample curves. This ELISA was validated according to the International Council for Harmonization Guidance. The inter-assay coefficient of variation was $\leq 30 \%$ for all antigens and secondary antibody combinations.

\section{Statistical Analysis}

Fisher's exact test was used to analyze the difference of rates for Mcat NP colonization, AOM and NP colonization progressing to AOM. The Mann-Whitney test was used to compare differences in Mcat protein-specific IgG or IgA concentrations, normalized by total IgG or IgA concentration in nasal wash, respectively. $P$ values of $<0.05$ were considered significant.

\section{RESULTS}

\section{Rates of Mcat NP Colonization and Percentages of Children with Mcat- Caused AOM in SOP and NOP Children}

Nasopharyngeal colonization of otopathogens is the first step in pathogenesis of AOM (23). We analyzed the rates of Mcat NP colonization in $68 \mathrm{sOP}$ and $560 \mathrm{NOP}$ children and assessed the percentage of children with Mcat-caused AOM in each population (Figure 1). Mcat NP colonization rates were consistently higher in sOP vs. NOP children: 33 vs. $27 \%$ at age 6 months, 31 vs. $25 \%$ at age 9 months, 34 vs. $26 \%$ at age 12 months, 26 vs. $23 \%$ at age 15 months, 26 vs. $23 \%$ at age 18 months, 36 vs. $22 \%$ at age 24 months $(P=0.010), 23$ vs. $11 \%$ at age $30-36$ months $(P=0.006)$, and overall 30 vs. $22 \%$ at age $6-36$ months old $(P=0.0003$, Figure 1A). The relative risk of Mcat NP colonization for sOP:NOP children was 1.7 at age 24 months old, 2.1 at age 30-36 months old, and overall 1.3-fold higher for age 6-36 months old.

Thirty seven of 75 (49\%) sOP children with AOM had Mcatcaused AOM, which is significantly higher than 62 of 255 (24\%) NOP children with AOM $(P<0.0001$, Figure 1B). The relative risk of Mcat-caused AOM for sOP:NOP was thus twofold higher.
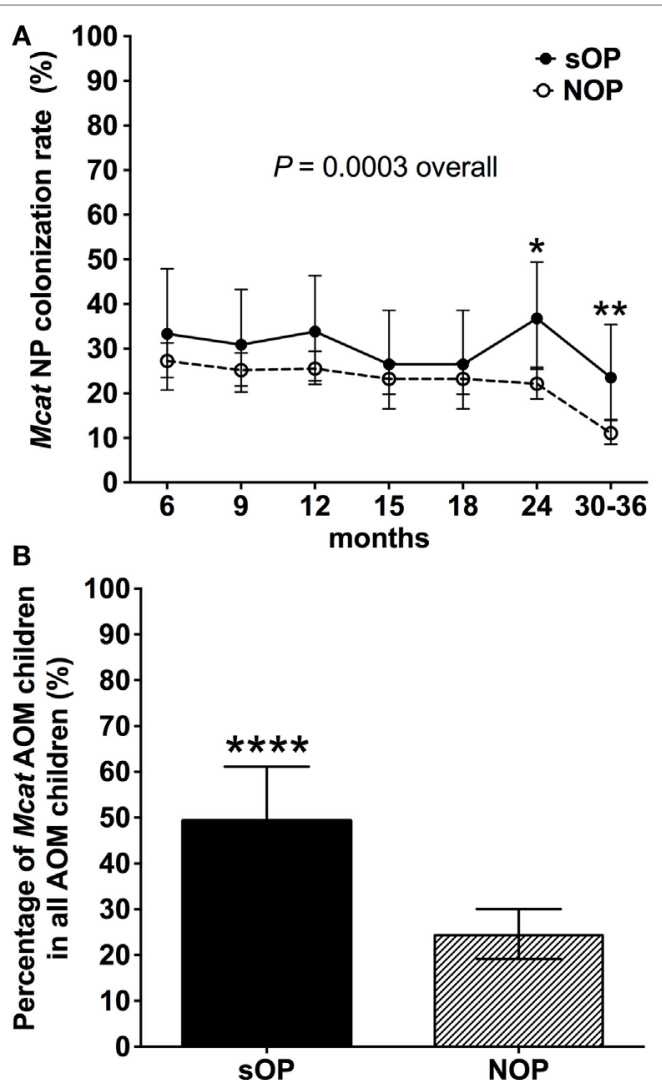

FIGURE 1 | Moraxella catarrhalis (Mcat) nasopharyngeal (NP) colonization rates and percentages of Mcat-caused acute otitis media (AOM) in stringently defined otitis prone (sOP) and non-otitis prone (NOP) children. (A) $60 \mathrm{sOP}$ children and 560 NOP children were assessed for Mcat NP colonization rates at $6,9,12,15,18,21,24$, and 30-36 months old, respectively. (B) 75 sOP children and 255 NOP children with AOM history were assessed for the percentage of children with Mcat-caused AOM. Data are represented as percentage $\pm 95 \%$ confidence interval. Fisher's exact test was used to compare the difference of rates or percentages between sOP and NOP children. ${ }^{\star} P<0.05,{ }^{\star \star} P<0.01$, and ${ }^{\star \star \star \star} P<0.0001$ comparing sOP vs. NOP children.

\section{Mucosal Antibody Levels Against Mcat Proteins in sOP and NOP Children}

We analyzed mucosal IgG and IgA antibodies in nasal wash samples from 238 healthy visits of 59 sOP children and 270 healthy visits of 75 NOP children age 6-36 months old. sOP children had significantly lower levels of mucosal IgG to OMP CD $(P<0.0001)$, OppA $(P=0.018)$, and Hag5-9 $(P<0.0001)$ than NOP children (Figure 2A). Similarly, sOP children had significantly lower levels of mucosal IgA antibody to Hag5-9 $(P<0.0001)$ and PilA2 $(P<0.0001)$ than NOP children (Figure 2B). We did not find a significant difference of mucosal IgG and IgA to the four Mcat proteins among different age time points $6,9,12,15,18,24$, and $30-36$ months in either sOP or NOP children $(P>0.05)$. Mucosal IgG levels to four proteins could be ranked as OMP CD $=$ OppA $=$ Hag5 $-9>$ PilA2 $(P<0.0001)$ in sOP and NOP children altogether. Mucosal IgA levels to the four proteins could be ranked as Hag5-9 > OMP 

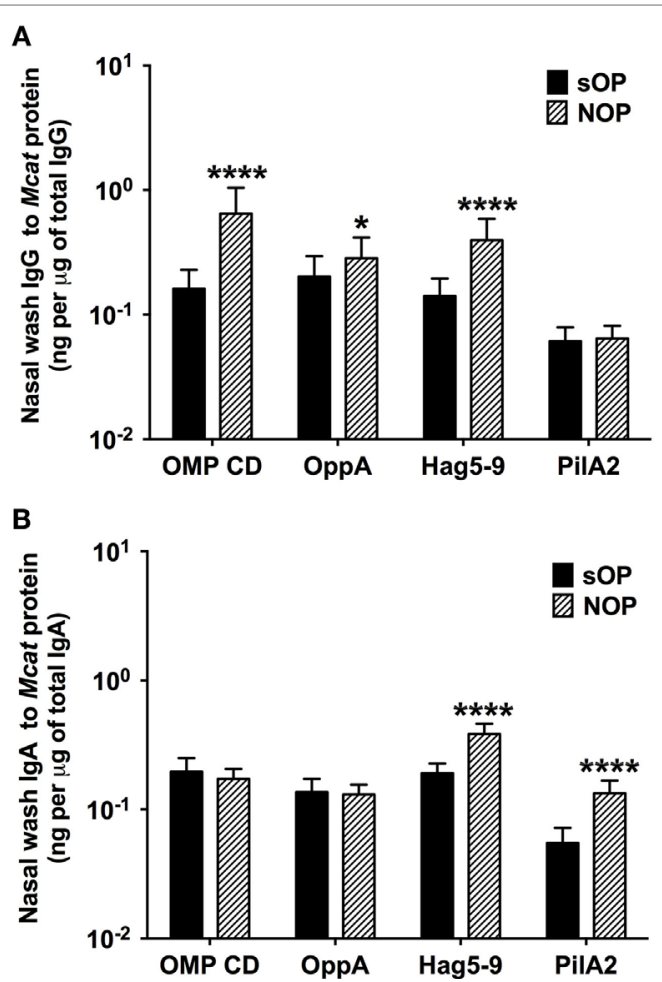

FIGURE 2 | Mucosal antibody levels against Moraxella catarrhalis (Mcat) proteins in stringently defined otitis prone (sOP) and non-otitis prone (NOP) children. Nasal wash IgG and IgA against Mcat proteins outer membrane protein (OMP) CD, oligopeptide permease A (OppA), Hag5-9, and Pilin A clade 2 (PilA2) were detected by using enzyme-linked immunosorbent assay for children age 6-36 months old. Mcat protein-specific (A) $\lg G$ and (B) $\lg A$ in nasal wash were compared between 238 healthy visits of 59 sOP children and 270 healthy visits of 75 NOP children. IgG and lgA concentrations (ng/ $\mathrm{ml})$ were normalized with total lgG and lgA concentrations $(\mu \mathrm{g} / \mathrm{ml})$ in nasal wash, respectively, for comparison. Data are represented as geometric mean $\pm 95 \%$ confidence interval. Mann-Whitney test was used for comparisons. ${ }^{\star} P<0.05$ and ${ }^{\star \star \star \star} P<0.0001$ comparing sOP vs. NOP children.

CD $>$ OppA $>$ PilA2 $(P<0.0001)$ in sOP and NOP children altogether (Figure 2).

\section{Mucosal Antibodies to Mcat Proteins at Healthy Visits with vs. without NP Colonization in sOP and NOP Children}

We compared the association of NP colonization status with mucosal antibody responses between sOP and NOP children at age 6-36 months old. We analyzed 107 healthy visits with positive Mcat NP colonization and 131 healthy visits with negative Mcat NP colonization for 59 sOP children. sOP children did not show a difference of mucosal IgG (Figure 3A) or IgA (Figure 3B) to any of the four protein antigens comparing Mcat NP colonization positive and negative visits. We also analyzed 130 healthy visits with Mcat NP colonization positive and 140 healthy visits with Mcat NP colonization negative for 75 NOP children. In contrast, NOP children without Mcat NP colonization had significantly higher mucosal IgG to Hag5-9 $(P=0.039)$ and PilA2 $(P=0.0076)$ than NOP children with Mcat NP colonization (Figure 3C). NOP children without Mcat NP colonization displayed a significantly higher mucosal IgA to OMP CD $(P=0.010)$, OppA $(P=0.030)$, and PilA2 $(P=0.043)$ than NOP children with Mcat NP colonization (Figure 3D).

In addition, mucosal IgG and IgA to Mcat proteins were examined for 50 healthy visits of 22 NOP children currently not colonized by Mcat but with Mcat colonization history and compared to those for 90 healthy visits of 25 NOP children who had never been colonized by Mcat (Figure S1 in Supplementary Material). We did not find a significant difference of mucosal antibody levels between these two groups of children $(P>0.05$, Figure S1 in Supplementary Material). Two out of 22 (9\%) NOP children currently not colonized by Mcat but with Mcat NP colonization history experienced one episode of Mcat-caused AOM whereas 3 out of 25 (12\%) of the NOP children never colonized by Mcat experienced one to two episodes of Mcat-caused AOM. There was no difference of Mcat-caused AOM rate between the two groups of children $(P>0.05)$. We did not find a difference of Mcat protein-specific mucosal antibodies between the children experiencing $\mathrm{AOM}$ and those without experiencing $\mathrm{AOM}$ in either group of NOP children $(P>0.05)$. Therefore, prior $M c a t$ NP colonization did not have a significant effect on mucosal antiMcat protein antibody levels and Mcat-caused AOM frequency in the NOP children without current Mcat NP colonization; however, the statistical power to detect differences was limited by the samples size of subjects and colonization/AOM events.

\section{Mucosal Antibodies to Mcat Proteins at Healthy Visits with Mcat NP Colonization vs. AOM Visits in SOP and NOP Children}

Moraxella catarrhalis NP colonization is an immunizing event producing systemic and mucosal antibodies which may confer protection against subsequent NP colonization and AOM infections. We compared the mucosal antibodies at asymptomatic colonization by Mcat vs. onset of Mcat-caused AOM to analyze the association of natural antibody induction with protection against AOM in both sOP and NOP children. Mucosal IgG and IgA were analyzed for 107 and 130 Mcat NP colonization visits of 47 sOP and 61 NOP children, respectively, and 42 and 43 Mcatcaused AOM visits of $31 \mathrm{sOP}$ and 37 NOP children, respectively. sOP children did not show a higher mucosal IgG (Figure 4A) or IgA (Figure 4B) to any of the four Mcat proteins at Mcat NP colonization compared to those at onset of AOM except a higher mucosal IgG to Hag5-9 upon Mcat NP colonization than that at onset of $\operatorname{AOM}(P=0.015$, Figure 4A). In contrast, NOP children displayed higher mucosal IgG to OMP CD $(P=0.0070$, Figure 4C) and Hag5-9 $(P=0.0003$, Figure 4C) and IgA to Hag5-9 $(P=0.0067$, Figure 4D) at Mcat NP colonization than that at onset of AOM. There was a significantly higher rate of Mcat NP colonization progressing to AOM in sOP children (28 in total of 107 Mcat NP colonization positive visits, 26\%) than that in NOP children (4 in total of 130 Mcat NP colonization positive visits, 3\%) $(P<0.0001$, Figure 5). The relative risk of asymptomatic colonization progressing to AOM for sOP was 8.5-fold higher than NOP children. 

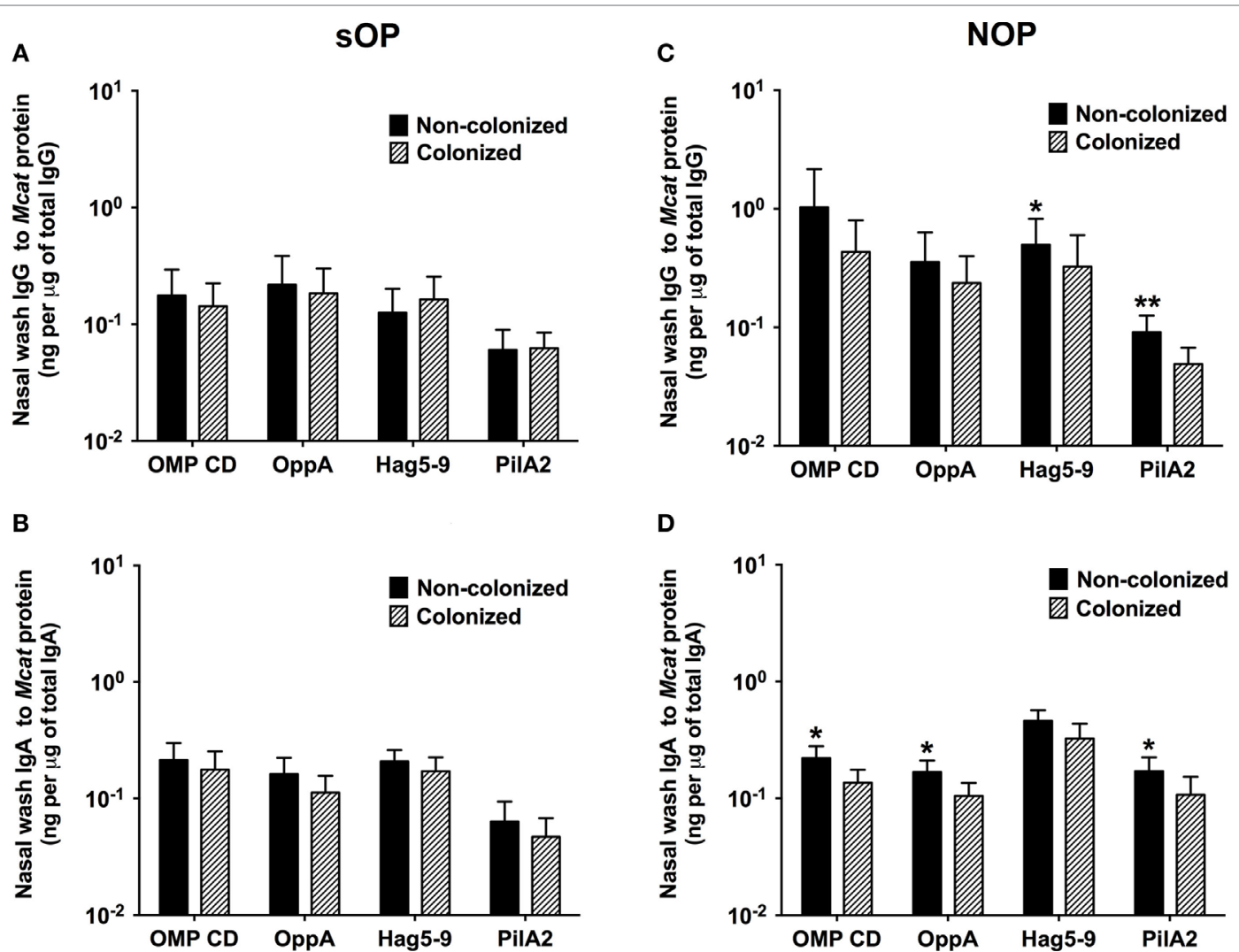

FIGURE 3 | Comparison of mucosal antibodies to Moraxella catarrhalis (Mcat) proteins between visits with and without Mcat nasopharyngeal (NP) colonization in stringently defined otitis prone (SOP) and non-otitis prone (NOP) children. Nasal wash IgG and lgA against Mcat proteins outer membrane protein (OMP) CD, oligopeptide permease A (OppA), Hag5-9, and Pilin A clade 2 (PilA2) were detected by using enzyme-linked immunosorbent assay for children age 6-36 months old. (A,B) 107 healthy visits with Mcat NP colonization positive and 131 healthy visits with Mcat NP colonization negative of 59 sOP children were analyzed for nasal wash (A) IgG and (B) IgA. (C,D) 130 healthy visits with Mcat NP colonization positive and 140 healthy visits with Mcat NP colonization negative of 75 NOP children were analyzed for nasal wash (C) lgG and (D) IgA. Mcat protein-specific lgG and lgA concentrations (ng/ml) were normalized with total lgG and lgA concentrations $(\mu \mathrm{g} / \mathrm{ml})$ in nasal wash, respectively, for comparison. Data are shown as geometric mean $\pm 95 \%$ confidence interval. Mann-Whitney test was used for comparison. ${ }^{\star} P<0.05$ and ${ }^{* \star} P<0.01$ comparing Mcat NP non-colonized vs. colonized visits in the same population of children.

\section{DISCUSSION}

Otitis prone is a health condition of frequent recurrence of AOM in children, usually younger than 5 years old. In this study, we found that sOP children displayed more frequent AOMs and asymptomatic NP colonizations caused by Mcat than NOP children, which is consistent with our prior observations on Mcat as well as otopathogens Spn and NTHi (24). Our previous study of children enrolled during years 2006-2011 showed that sOP children had a higher Mcat NP colonization rate than NOP children at age 6-12 months old (24). However, when we included more subjects enrolled during years 2011-2016, sOP children exhibited higher Mcat NP colonization rates than NOP children at a later age phase of 24-36 months old, which suggests that Mcat NP colonization trends to be more prevalent in older sOP children during more recent years. Indeed, Mcat has been considered a less virulent bacterium than Spn or NTHi and has not been associated with TM rupture or severe illness in young children (25). However, during our 10-year (2006-2016) prospective, longitudinal clinical, and translational studies of AOM, only since 2013 have we observed cases of severe AOM symptoms and TM rupture caused by Mcat (Pichichero et al., unpublished observations), suggesting that the virulence of Mcat as an otopathogen may be increasing. Mcat causing TM rupture was first described in Israel in 2009 (25) and in Italy in 2017 (26), suggesting the emergence of more virulent Mcat is a potentially significant problem of global proportions. Also, Mcat NP colonization rates have significantly increased since the introduction of pneumococcal glycoconjugate vaccines in children (27). The pattern of Mcat NP colonization increase in our study may be due to the higher prevalence of emerging virulent Mcat strains in older sOP children age 24-36 months.

Studies have shown that AOM has a multifactorial etiology (4). The mechanism of OP has been attributed to many pathogenic factors, such as preceding upper respiratory infection, immature Eustachian tube, social and environmental risk factors (day care environment, parent smoking, multiple siblings at home, etc.) (4), and importantly the delayed maturation of the child's immune systems as identified by our group (15). In this study, we observed decreased mucosal IgG and IgA responses to all four Mcat proteins in sOP children at healthy visits, which is 

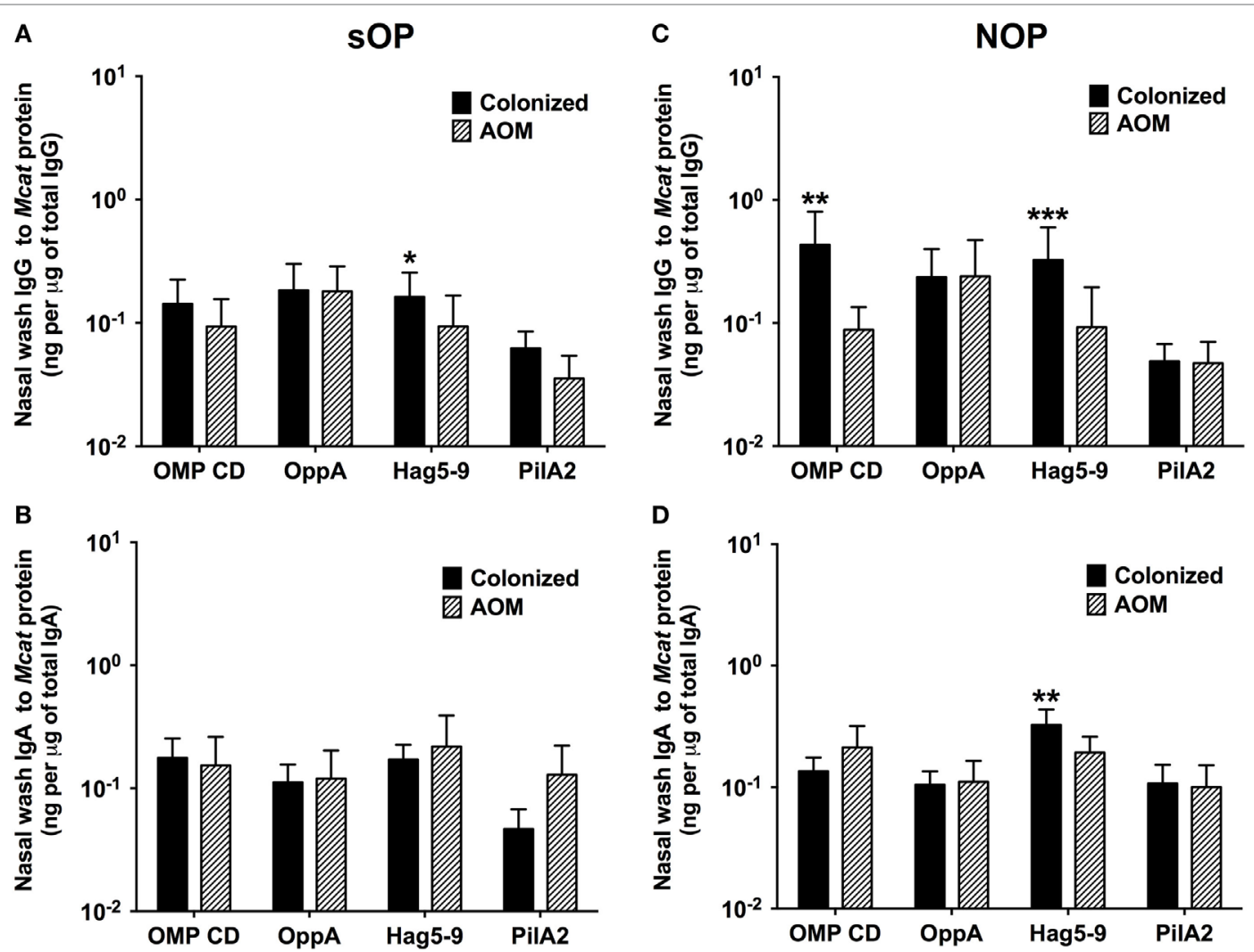

FIGURE 4 | Mucosal antibodies to Moraxella catarrhalis (Mcat) proteins at healthy visits with Mcat nasopharyngeal (NP) colonization vs. acute otitis media (AOM) visits in stringently defined otitis prone (SOP) and non-otitis prone (NOP) children. Nasal wash IgG and lgA against Mcat proteins outer membrane protein (OMP) CD, oligopeptide permease A (OppA), Hag5-9, and Pilin A clade 2 (PilA2) were detected by using enzyme-linked immunosorbent assay for children age 6-36 months old. (A,B) 107 Mcat NP colonization visits of 47 sOP children and 42 Mcat-caused AOM visits of 31 sOP children were analyzed for nasal wash (A) lgG and (B) lgA. (C,D) 130 Mcat NP colonization visits of 61 NOP children and 43 Mcat-caused AOM visits of 37 NOP children were analyzed for nasal wash (C) IgG and (D) IgA. Mcat protein-specific lgG and lgA concentrations ( $\mathrm{ng} / \mathrm{ml}$ ) were normalized with total lgG and lgA concentrations ( $\mu \mathrm{g} / \mathrm{ml})$ in nasal wash, respectively, for comparison. Data are shown as geometric mean $\pm 95 \%$ confidence interval. Mann-Whitney test was used for comparison. ${ }^{\star} P<0.05$, ${ }^{\star \star} P<0.01$ and ${ }^{\star \star \star} P<0.001$ comparing Mcat NP colonized vs. AOM visits in the same population of children.

in concert with our prior observations on diminished serum and/ or mucosal antibody responses to Spn $(17,24)$, NTHi (18), and Mcat (Ren et al., unpublished) protein antigens in this population. In accordance with our prior findings on serum antibody responses (16), high mucosal antibodies correlated with low Mcat NP carriage, suggesting protection by naturally acquired Mcat protein-specific mucosal antibodies in NOP but not sOP children. Verhaegh and coworkers had similar findings. They showed serum IgG levels to MID962-1200 were higher in 2-yearold children without Mcat NP colonization compared to children with Mcat NP colonization (28).

Age of the child and preexisting antibody levels are important covariates in predicting an antibody response to NP colonization. Our prior studies showed varied serum IgG responses during NP colonization of Spn and NTHi vs. non-NP colonization in children age 6-30 months old (19, 20, 24). Spn and NTHi also display differences from Mcat in producing acute responses to AOM (29). Thus, different pathogens may evoke different patterns of antibody responses in relation to NP colonization in young children, reinforcing the need for systematic study of each organism in different hosts. However, consistent with our prior observations on antibody responses to Spn and NTHi, sOP children tend to produce lower mucosal antibody responses to natural exposure to Mcat proteins than NOP children suggestive of a more neonatal-like, delayed immune maturation response.

Mucosal antibodies to $\operatorname{OMP} \operatorname{CD}(22,30)$ and Hag $(9,31)$ have been detected in healthy adults and in those with chronic obstructive pulmonary disease. Mucosal antibodies to Hag have also been observed in young children (32). The current results show that all four Mcat protein antigens are immunogenic with regard to mucosal antibodies in young children. The mucosal IgG levels to four Mcat proteins could be ranked as $\mathrm{OMP} \mathrm{CD}=\mathrm{OppA}=\mathrm{Hag} 5-9>\mathrm{PilA} 2$ while mucosal IgA levels to four Mcat proteins could be ranked as Hag5-9 > OMP $\mathrm{CD}>\mathrm{OppA}>\mathrm{PilA} 2$ in both sOP and NOP children. The current mucosal data support our prior serum data that OMP CD, OppA, and Hag5-9 exhibit promising immunogenicity for Mcat vaccine candidates (16).

Nasopharyngeal colonization of pathogens induces naturally acquired serum and mucosal antibodies, which may confer 


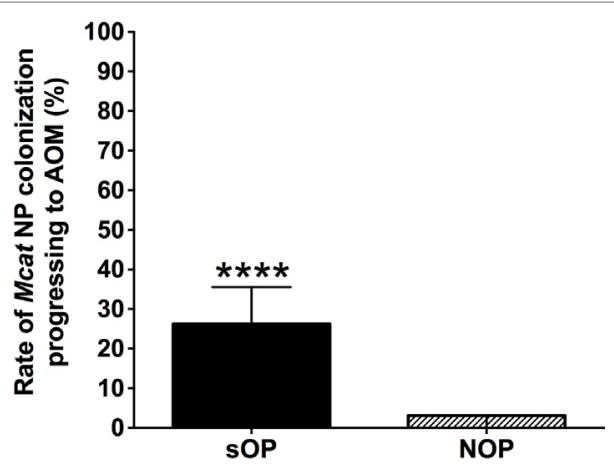

FIGURE 5 | Rates of Moraxella catarrhalis (Mcat) nasopharyngeal (NP) colonization progressing to acute otitis media (AOM) in stringently defined otitis prone (SOP) and non-otitis prone (NOP) children. Rates of Mcat NP colonization progressing to Mcat-caused AOM were assessed for $47 \mathrm{sOP}$ and 61 NOP children age 6-36 months old. Data are represented as percentage $\pm 95 \%$ confidence interval $(\mathrm{Cl})$. Ninety five percent $\mathrm{Cl}$ (error bar) for NOP children cannot be calculated by Fisher's exact test due to only four (less than five) episodes of Mcat NP colonization progressing to AOM in NOP children. Fisher's exact test was used to compare the difference in the rates between sOP and NOP children. ${ }^{* \star \star \star} P<0.0001$ comparing sOP vs. NOP children.

host defense against infections. The current mucosal results are consistent with our prior serum data that high antibodies to Mcat proteins are associated with reduced Mcat carriage, suggesting a protective effect of naturally induced antibodies against NP colonization. The observation of reduced mucosal antibodies in the current study is in line with our prior data of decreased serum antibodies to Mcat proteins in sOP children during asymptomatic colonization, suggesting that sOP children had a deficient immune responses to Mcat. Stenfors and Raisanen found that there are less secretory $\operatorname{IgA}(\operatorname{sg} \mathrm{A})$-coated bacteria in the nasopharynx of OP children, which may be responsible for the otitis-prone condition (33). Our results of lower naturally acquired mucosal Mcat-specific IgA support Stenfors' findings in OP children. Impaired mucosal IgA production could lead to less inhibition of Mcat adherence to NP epithelial cells and higher NP colonization in sOP children, which was observed in both the current and prior studies $(24,34)$.

High frequency of NP colonization of otopathogens Spn, $\mathrm{NTHi}$, and Mcat is associated with increased development of AOM (35). Among the three otopathogens, early colonization with Mcat is associated with the greatest risk of AOM and otitis media with effusion (35). Our results had consistent findings for sOP children of lower production of mucosal IgG and IgA to Mcat proteins upon NP colonization and a higher rate of progressing to Mcat-caused AOM. On the other hand, NOP children had higher mucosal antibodies to Mcat proteins and lower rates of $\mathrm{NP}$ colonization progressing to AOM ( $>8$ times lower than sOP children). Mucosal immunity is the frontline of host defense against bacterial infections. Enhanced bactericidal effect of IgG and coating of bacteria with sIgA to inhibit bacterial adherence to respiratory epithelia may account for the protection against AOM observed in this study.
In summary, sOP children had higher NP colonization of Mcat, Mcat-caused AOM rates and a higher percentage of Mcat NP colonization progressing to Mcat-caused AOM than NOP children. These pathogenesis events and outcomes correlate with impaired mucosal antibody responses to Mcat protein antigens. Mucosal vaccination with immunogenic Mcat antigens and developing mucosal adjuvants should be pursued for preventing AOM caused by Mcat, with special attention to the sOP child.

\section{AUTHOR CONTRIBUTIONS}

DR and MP conceived and designed the study. DR, MP, TM, and EL provided materials. DR performed experiments and statistical analysis. DR and MP wrote the manuscript. All the authors have approved the final manuscript.

\section{ACKNOWLEDGMENTS}

The authors are grateful to Dr. Anthony A. Campagnari and Dr. Nicole Luke-Marshall at University at Buffalo, The State University of New York for their kindness to provide the expression plasmid and bacterial strains for production of PilA2 recombinant protein. The authors are indebted to Jill Mangiafesto and Konnor Shares for their ELISA work. The authors thank Dr. Janet Casey, the nurses and staff of Legacy Pediatrics, the collaborating pediatricians from Sunrise Pediatrics, Westfall Pediatrics, Lewis Pediatrics, and Long Pond Pediatrics, and the parents who consented and the children who participated in this challenging study. The authors also thank the support of materials and methods from R01 DC012200 to TM.

\section{FUNDING}

This work was supported by NIH NIAID R03 AI113649 to DR and NIDCD R01 DC008671 to MP. The funders had no role in study design, data collection and analysis, decision to publish, or preparation of the manuscript.

\section{SUPPLEMENTARY MATERIAL}

The Supplementary Material for this article can be found online at http://journal.frontiersin.org/article/10.3389/fimmu.2017.00953/ full\#supplementary-material.

FIGURE S1 | Comparison of mucosal antibodies to Moraxella catarrhalis (Mcat) proteins between visits with and without Mcat nasopharyngeal (NP) colonization history in non-otitis prone (NOP) children without current Mcat NP colonization. Nasal wash IgG and IgA against Mcat proteins outer membrane protein (OMP) CD, oligopeptide permease A (OppA), Hag5-9, and Pilin A clade 2 (PilA2) were detected by using enzyme-linked immunosorbent assay for children age 6-36 months old. Mcat protein-specific (A) $\lg G$ and (B) $\operatorname{lgA}$ in nasal wash were compared between 50 healthy visits of 22 NOP children currently not colonized by Mcat but with Mcat colonization history and 90 healthy visits of $25 \mathrm{NOP}$ children never colonized by Mcat. IgG and $\operatorname{lgA}$ concentrations $(\mathrm{ng} / \mathrm{ml})$ were normalized with total lgG and $\lg A$ concentrations $(\mu \mathrm{g} / \mathrm{ml})$ in nasal wash, respectively, for comparison. Data are represented as geometric mean $\pm 95 \%$ confidence interval. Mann-Whitney test was used for comparisons. 


\section{REFERENCES}

1. Casey JR, Kaur R, Friedel VC, Pichichero ME. Acute otitis media otopathogens during 2008 to 2010 in Rochester, New York. Pediatr Infect Dis J (2013) 32(8):805-9. doi:10.1097/INF.0b013e31828d9acc

2. Casey JR, Kauer R, Pichichero M. Otopathogens causing otitis media in the 13-valent pneumococcal conjugate vaccine era. 18th International Symposium on Recent Advances in Otitis Media. Oxon Hill (2015). 211 p.

3. Sillanpaa S, Oikarinen S, Sipila M, Kramna L, Rautiainen M, Huhtala H, et al. Moraxella catarrhalis might be more common than expected in acute otitis media in young Finnish children. J Clin Microbiol (2016) 54(9):2373-9. doi:10.1128/JCM.01146-16

4. Schilder AG, Chonmaitree T, Cripps AW, Rosenfeld RM, Casselbrant ML, Haggard MP, et al. Otitis media. Nat Rev Dis Primers (2016) 2:16063. doi:10.1038/nrdp.2016.63

5. Holm MM, Vanlerberg SL, Foley IM, Sledjeski DD, Lafontaine ER. The Moraxella catarrhalis porin-like outer membrane protein $\mathrm{CD}$ is an adhesin for human lung cells. Infect Immun (2004) 72(4):1906-13. doi:10.1128/ IAI.72.4.1906-1913.2004

6. Murphy TF, Kirkham C, Lesse AJ. The major heat-modifiable outer membrane protein CD is highly conserved among strains of Branhamella catarrhalis. Mol Microbiol (1993) 10(1):87-97. doi:10.1111/j.1365-2958.1993.tb00906.x

7. Yang M, Johnson A, Murphy TF. Characterization and evaluation of the Moraxella catarrhalis oligopeptide permease A as a mucosal vaccine antigen. Infect Immun (2011) 79(2):846-57. doi:10.1128/IAI.00314-10

8. Jones MM, Johnson A, Koszelak-Rosenblum M, Kirkham C, Brauer AL, Malkowski MG, et al. Role of the oligopeptide permease $\mathrm{ABC}$ transporter of Moraxella catarrhalis in nutrient acquisition and persistence in the respiratory tract. Infect Immun (2014) 82(11):4758-66. doi:10.1128/IAI.02185-14

9. LaFontaine ER, Snipes LE, Bullard B, Brauer AL, Sethi S, Murphy TF. Identification of domains of the Hag/MID surface protein recognized by systemic and mucosal antibodies in adults with chronic obstructive pulmonary disease following clearance of Moraxella catarrhalis. Clin Vaccine Immunol (2009) 16(5):653-9. doi:10.1128/CVI.00460-08

10. Luke NR, Howlett AJ, Shao J, Campagnari AA. Expression of type IV pili by Moraxella catarrhalis is essential for natural competence and is affected by iron limitation. Infect Immun (2004) 72(11):6262-70. doi:10.1128/ IAI.72.11.6262-6270.2004

11. Luke-Marshall NR, Sauberan SL, Campagnari AA. Comparative analyses of the Moraxella catarrhalis type-IV pilus structural subunit PilA. Gene (2011) 477(1-2):19-23. doi:10.1016/j.gene.2011.01.010

12. Luke NR, Jurcisek JA, Bakaletz LO, Campagnari AA. Contribution of Moraxella catarrhalis type IV pili to nasopharyngeal colonization and biofilm formation. Infect Immun (2007) 75(12):5559-64. doi:10.1128/IAI.00946-07

13. Poehling KA, Szilagyi PG, Grijalva CG, Martin SW, LaFleur B, Mitchel E, et al. Reduction of frequent otitis media and pressure-equalizing tube insertions in children after introduction of pneumococcal conjugate vaccine. Pediatrics (2007) 119(4):707-15. doi:10.1542/peds.2006-2138

14. Pichichero ME, Casey JR, Almudevar A. Reducing the frequency of acute otitis media by individualized care. Pediatr Infect Dis J (2013) 32(5):473-8. doi:10.1097/INF.0b013e3182862b57

15. Pichichero ME. Ten-year study of the stringently defined otitis-prone child in Rochester, NY. Pediatr Infect Dis J (2016) 35(9):1033-9. doi:10.1097/ INF.0000000000001217

16. Ren D, Almudevar AL, Murphy TF, Lafontaine ER, Campagnari AA, Luke-Marshall N, et al. Serum antibody response to Moraxella catarrhalis proteins OMP CD, OppA, Msp22, Hag, and PilA2 after nasopharyngeal colonization and acute otitis media in children. Vaccine (2015) 33(43):5809-14. doi:10.1016/j.vaccine.2015.09.023

17. Kaur R, Casey JR, Pichichero ME. Serum antibody response to five Streptococcus pneumoniae proteins during acute otitis media in otitis-prone and non-otitis-prone children. Pediatr Infect Dis J (2011) 30(8):645-50. doi:10.1097/INF.0b013e31821c2d8b

18. Kaur R, Casey JR, Pichichero ME. Serum antibody response to three nontypeable Haemophilus influenzae outer membrane proteins during acute otitis media and nasopharyngeal colonization in otitis prone and non-otitis prone children. Vaccine (2011) 29(5):1023-8. doi:10.1016/j.vaccine.2010.11.055

19. Pichichero ME, Kaur R, Casey JR, Sabirov A, Khan MN, Almudevar A. Antibody response to Haemophilus influenzae outer membrane protein D,
P6, and OMP26 after nasopharyngeal colonization and acute otitis media in children. Vaccine (2010) 28(44):7184-92. doi:10.1016/j.vaccine.2010.08.063

20. Pichichero ME, Kaur R, Casey JR, Xu Q, Almudevar A, Ochs M. Antibody response to Streptococcus pneumoniae proteins PhtD, LytB, PcpA, PhtE and Ply after nasopharyngeal colonization and acute otitis media in children. Hum Vaccin Immunother (2012) 8(6):799-805. doi:10.4161/hv.19820

21. Xu Q, Casey JR, Pichichero ME. Higher levels of mucosal antibody to pneumococcal vaccine candidate proteins are associated with reduced acute otitis media caused by Streptococcus pneumoniae in young children. Mucosal Immunol (2015) 8(5):1110-7. doi:10.1038/mi.2015.1

22. Murphy TF, Kirkham C, Liu DF, Sethi S. Human immune response to outer membrane protein $\mathrm{CD}$ of Moraxella catarrhalis in adults with chronic obstructive pulmonary disease. Infect Immun (2003) 71(3):1288-94. doi:10.1128/ IAI.71.3.1288-1294.2003

23. Casey JR, Adlowitz DG, Pichichero ME. New patterns in the otopathogens causing acute otitis media six to eight years after introduction of pneumococcal conjugate vaccine. Pediatr Infect Dis J (2010) 29(4):304-9. doi:10.1097/ INF.0b013e3181c1bc48

24. Xu Q, Casey JR, Newman E, Pichichero ME. Otitis-prone children have immunologic deficiencies in naturally acquired nasopharyngeal mucosal antibody response after Streptococcus pneumoniae colonization. Pediatr Infect Dis J (2016) 35(1):54-60. doi:10.1097/INF.0000000000000949

25. Broides A, Dagan R, Greenberg D, Givon-Lavi N, Leibovitz E. Acute otitis media caused by Moraxella catarrhalis: epidemiologic and clinical characteristics. Clin Infect Dis (2009) 49(11):1641-7. doi:10.1086/647933

26. Principi N, Marchisio P, Rosazza C, Sciarrabba CS, Esposito S. Acute otitis media with spontaneous tympanic membrane perforation. EurJ Clin Microbio Infect Dis (2017) 36(1):11-8. doi:10.1007/s10096-016-2783-9

27. Revai K, McCormick DP, Patel J, Grady JJ, Saeed K, Chonmaitree T. Effect of pneumococcal conjugate vaccine on nasopharyngeal bacterial colonization during acute otitis media. Pediatrics (2006) 117(5):1823-9. doi:10.1542/ peds.2005-1983

28. Verhaegh SJ, de Vogel CP, Riesbeck K, Lafontaine ER, Murphy TF, Verbrugh HA, et al. Temporal development of the humoral immune response to surface antigens of Moraxella catarrhalis in young infants. Vaccine (2011) 29(34):5603-10. doi:10.1016/j.vaccine.2011.06.019

29. Liu K, Pichichero ME. Clinical significance of serum S100A12 in acute otitis media in young children. Pediatr Infect Dis J (2012) 31(3):e56-8. doi:10.1097/ INF.0b013e31824672cb

30. Stutzmann Meier P, Heiniger N, Troller R, Aebi C. Salivary antibodies directed against outer membrane proteins of Moraxella catarrhalis in healthy adults. Infect Immun (2003) 71(12):6793-8. doi:10.1128/IAI.71.12.6793-6798.2003

31. Murphy TF, Brauer AL, Aebi C, Sethi S. Antigenic specificity of the mucosal antibody response to Moraxella catarrhalis in chronic obstructive pulmonary disease. Infect Immun (2005) 73(12):8161-6. doi:10.1128/ IAI.73.12.8161-8166.2005

32. Meier PS, Freiburghaus S, Martin A, Heiniger N, Troller R, Aebi C. Mucosal immune response to specific outer membrane proteins of Moraxella catarrhalis in young children. Pediatr Infect Dis J (2003) 22(3):256-62. doi:10.1097/01. inf.0000054827.86683.bd

33. Stenfors LE, Raisanen S. Secretory IgA-, IgG-and C3b-coated bacteria in the nasopharynx of otitis-prone and non-otitis-prone children. Acta Otolaryngol (1993) 113(2):191-5. doi:10.3109/00016489309135791

34. Stenfors LE, Raisanen S. Abundant attachment of bacteria to nasopharyngeal epithelium in otitis-prone children. J Infect Dis (1992) 165(6):1148-50. doi:10.1093/infdis/165.6.1148

35. Faden H, Duffy L, Wasielewski R, Wolf J, Krystofik D, Tung Y. Relationship between nasopharyngeal colonization and the development of otitis media in children. Tonawanda/Williamsville pediatrics. J Infect Dis (1997) 175(6):1440-5. doi:10.1086/516477

Conflict of Interest Statement: TM has patents for vaccines for $M$. catarrhalis. No other potential conflicts of interest were disclosed.

Copyright (๑) 2017 Ren, Murphy, Lafontaine and Pichichero. This is an open-access article distributed under the terms of the Creative Commons Attribution License (CC BY). The use, distribution or reproduction in other forums is permitted, provided the original author(s) or licensor are credited and that the original publication in this journal is cited, in accordance with accepted academic practice. No use, distribution or reproduction is permitted which does not comply with these terms. 